\title{
Bridge Named After the Mathematician Who Discovered the Chinese Remainder Theorem
}

\section{Xu Wenwen and Yu Ning}

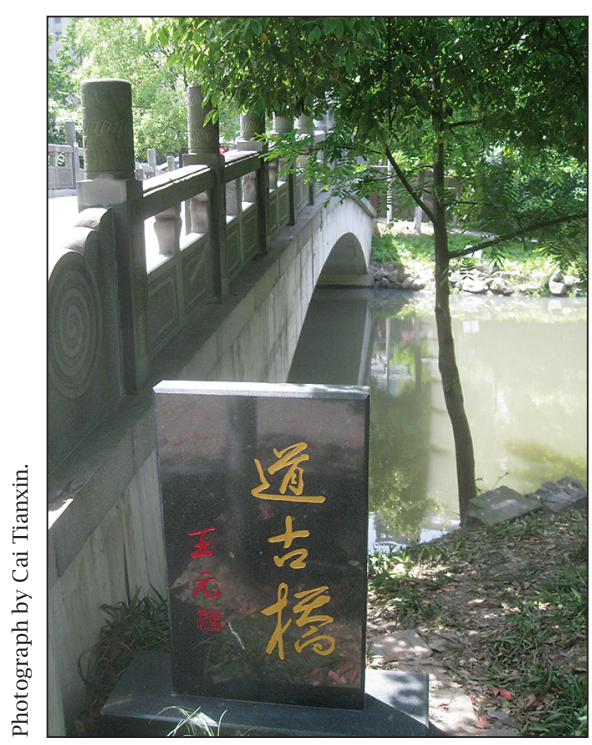

The Daogu Bridge, with a stone tablet at the side, was named after the ancient Chinese mathematician Qin Jiushao. secrets.

According to local historical records, the bridge was built between the years of 1237 and 1241. It was demolished a decade ago during a local building project. However, in 2005 a new stone bridge was built about 100 meters away from the original location over the river, with willow trees swaying

$\mathrm{Xu}$ Wenwen is a journalist with the Shanghai Daily. Her email address is xuwenwenhz@gmai 1 . com.

Yu Ning had an internship with the Shanghai Daily and is now an elementary school teacher in Ningbo City, Zhejiang Province. Her email address is 230289340@ sina.com.

DOI: http://dx.doi.org/10.1090/noti993 on both banks. It is now named Daogu Bridge, as Cai proposed.

"Though the site and bridge are now removed, its history and story remain," says Cai, who contributed to the renaming and attended the renaming ceremony held on the bridge on April 27. Cai says that historical gazetteers reveal that the old Daogu Bridge was named after a mathematician of the Southern Song Dynasty (1127-1279), Qin Jiushao, or spelled Ch'in Chiu-Shao (1208-1261) (Daogu is Qin's courtesy name).

Qin, regarded as one of the greatest mathematicians of the thirteenth century, created the worldrenowned Chinese Remainder Theorem, considered the most famous scientific theorem of ancient China. His reputation lies in the Mathematical Treatise in Nine Sections (Shu Shu Jiu Zhang), issued in 1247 , which covered matters that ranged from indeterminate analysis to military matters and surveying.

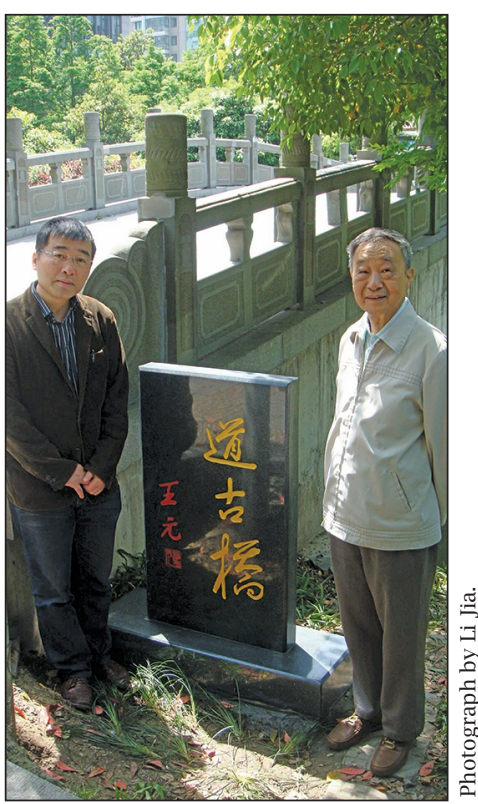

Cai Tianxin and Tang Xiaowei, academician, at the tablet ceremony at the Daogu Bridge. 


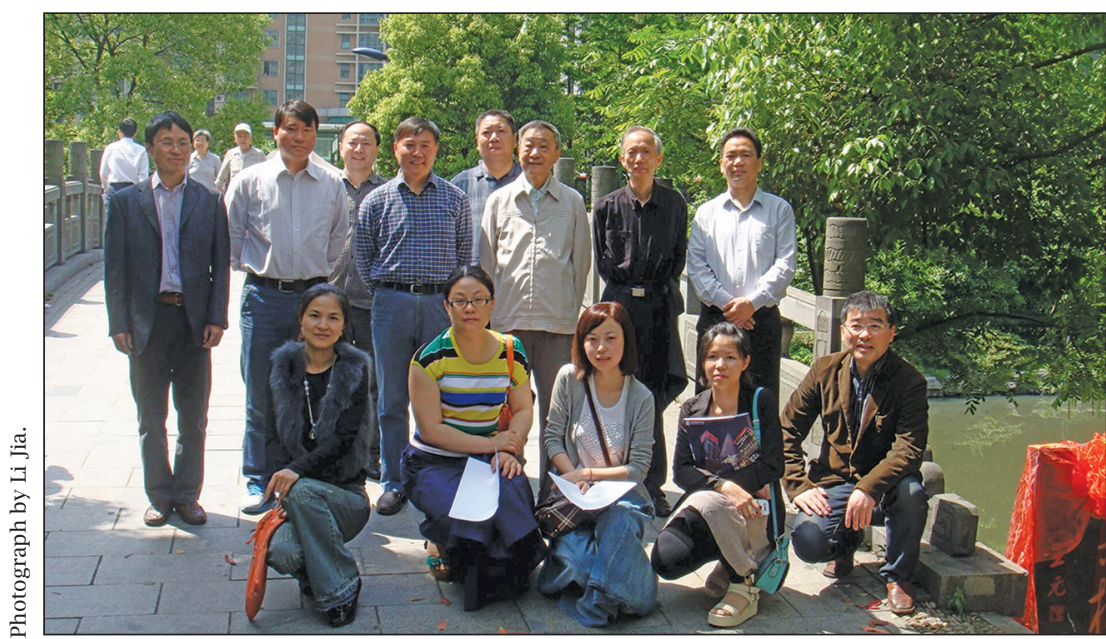

Guests and journalists at the tablet ceremony.

In the treatise, besides the Chinese Remainder Theorem, Qin also included "Qin Jiushao's formula" for finding the area of a triangle with given length of three sides, which is the same as Heron's formula, discovered earlier. No wonder the German mathematical historian M. Cantor praised him as "the luckiest genius," and American scientific historian George Sarton regarded him as one of the greatest scientists of the time.

Qin was born in Sichuan Province, and his ancestry can be traced back to Shandong. In 1219 he settled near the Xixi River in Hangzhou, a city nowadays only forty minutes away from Shanghai by bullet train, which was then known as Lin'an and was the dynastic capital when his father was assigned as an official there. "Hailed as a genius since his boyhood, he not only devoted his life to mathematics but also to many other fields, such as astronomy, engineering, and music, and even held a series of bureaucratic positions in several Chinese provinces," Cai says.

"In 1238, when Qin went back to Hangzhou for his father's funeral, the man found there was no bridge over the Xixi River, a fact which caused inconvenient transportation. As a result, the "Daogu Bridge" was built under his design as well as financial support from the local government. At the beginning the bridge was called Xixi Bridge for its location and was later altered to Daogu Bridge when Zhu Shijie, another famous mathematician from North China, traveled to Hangzhou in the early Yuan Dynasty (1271-1368) and proposed changing Xixi Bridge into Daogu Bridge in honor of Qin Jiushao.

"One reason that the bridge was forgotten and not even named after Qin is that the mathematician, who went into politics, was later, in articles written by two of his political opponents, described as a person who bribed and poisoned someone. However, centuries later in the Qing
Dynasty several scholars doubted these claims and tried to rehabilitate him. Today there remains no certain answer to Qin's political merit, and time has cast a shroud over the convoluted story."

A year ago, Cai, who is also a number theorist like Qin as well as an editor of the Hong Kong-based magazine Mathematical Culture, and who had lived near the bridge for over eighteen years and recently visited the place, was aware that the bridge is in similar surroundings to those of the original Daogu Bridge, which drove him to the idea of renaming the new bridge with the historic name. With Cai's endeavors and support from local government, a naming ceremony was officially held.

Beside the bridge there is a tablet with the inscription "Daogu Bridge", written by eightythree-year-old Wang Yuan, a renowned Chinese mathematician, educator, and popular science writer. On the reverse side of the tablet an article tells the historic story.

The site of the bridge is not exactly a scenic attraction, yet the city of Hangzhou is known as a tourist's paradise: the city's West Lake was inscribed on the list of World Heritage Sites, and the Xixi Wetland, which is not far from the bridge, is also a must-visit location. 